\title{
"Graue Eminenzen und schiefe Existenzen": Die deutschsprachigen Berater der Alliierten in Argentinien während des Zweiten Weltkrieges
}

\author{
Ronald C. Newton
}

Der Gegenstand der nachstehenden Ausführungen stellt einen Teil einer wesentlich umfangreicheren Arbeit dar, die kürzlich unter dem Titel The 'Nazi Menace' in Argentina, 1931-1947 in Nordamerika erschienen ist (Newton 1992). Diese Studie hat in erster Linie zwei Themen: Zum einen die Aktivitäten der Deutschen in Argentinien in den 30er und 40er Jahren, die Aktivitäten der diplomatischen Mission des Dritten Reiches, der Nazis und ihrer Mitläufer ebenso wie die der deutschsprachigen Flüchtlinge und des antifaschistischen Widerstandes. Insofern handelt es sich hier um eine Arbeit der Synthese, die sich auf jüngere Forschungen anderer Wissenschaftler bezieht, unter anderem auch solcher, die in diesem Band vertreten sind.

Der andere Themenbereich umfaßt eine Untersuchung der Rivalitäten zwischen den großen Mächten im selben Zeitraum, mit anderen Worten: des Kampfes um Einfluß und/oder Vorherrschaft zwischen Hitler-Deutschland, Großbritannien, den Vereinigten Staaten und nicht zuletzt Argentinien selbst. Die internationale Situation war außerordentlich komplex; jedoch war der zweifellos bitterste und verhängnisvollste Konflikt der zwischen Deutschland und den USA. Kurz gesagt: Die amerikanische Regierung unter Franklin D. Roosevelt nutzte den Einsatz der Hitler-Deutschen in Argentinien, der eine wenngleich in böser Absicht verstandene - Realität war, als Gelegenheit, den gesamten Einfluß der Deutschen aus dem Lande zu drängen. Und diese Kampagne Washingtons diente einem noch viel weiter gesteckten Ziel, nämlich alle auswärtigen Mächte unter Einschluß Großbritanniens aus der westlichen $\mathrm{He}$ misphäre zu verdrängen, um dort ein für alle Male die amerikanische Hegemonie zu etablieren. Insofern diese Politik, wie dies in der Regel geschieht, von Washington nicht einmal sich selber eingestanden wurde, war die öffentliche Debatte hinsichtlich der argentinischen Angelegenheiten durch ein hohes Maß an Weltfremdheit, Heuchelei und Lügen charakterisiert; erst seit den 70er Jahren haben Historiker in einigen Ländern die Vorstellungen hinsichtlich Argentiniens in den 30 er und 40er Jahren klären und berichtigen können. Und wie es dann kam, erwies sich die große Nazi-Bedrohung als höchst nützliches Hirngespinst.

Die beiden Themenbereiche dieser Arbeit berühren sich an dem Punkt, an dem die Alliierten es für nötig hielten, Informationen und Ratschläge über deutsche Motive und Fähigkeiten zu erhalten. In dieser weltweiten Notlage machten Briten und Amerikaner ausgiebigen Gebrauch von den am La Plata gestrandeten Flüchtlingen. Diese Flüchtlinge umfaßten ein immens breites 
Spektrum, von den ernsthaftesten und ehrenhaftesten Persönlichkeiten bis hin zu den übelsten Schurken. Normalerweise wollten die Nordamerikaner nichts mit links orientierten Deutschen zu tun haben - und umgekehrt. ${ }^{1}$ Sie wandten sich vielmehr an Nicht-Marxisten unter Einschluß von Liberalen, aber auch an Abenteurer, Scharlatane und Kriminelle. Fünf Beispiele sollen dies belegen.

Die "Schwarze Front", jene radikalen Nazis, die sich seit dem sog. RöhmPutsch und der "Säuberung" von Gregor Strasser, Ernst Röhm sowie der gesamten SA-Führung im Juli 1934 mit Hitler überworfen hatten, war die am wenigsten ansehnliche Erscheinung des antifaschistischen Widerstandes (von zur Mühlen 1985, 143-157). Ihr Gründer in Südamerika, ihre treibende Kraft und das Bindeglied zwischen dem argentinischen, britischen und amerikanischen Geheimdienst und - im Jahre 1942 - Führer und vielleicht einziges Mitglied einer "Deutschen Legion", die sich bei den Alliierten um die Billigung als Polizeitruppe in Nachkriegsdeutschland bemühte, war Bruno Fricke. Fricke durchlief seine Lehrzeit als politischer Desperado in verschiedenen Freikorps unter Einschluß der Organisation "Konsul" und der Roßbach-Gruppe unmittelbar nach dem Ersten Weltkrieg. Sein Deckmantel in der Roßbach-Gruppe war der eines Privatdetektivs (für kriminelle und Scheidungsangelegenheiten); ebenso war er in Waffenschmuggel verstrickt. Im Juni 1923 wurde er wegen Anstiftung zur Vertuschung eines politischen Mordes an einem gewissen Walter Cadow angeklagt. 1928 hielt er sich in Paraguay auf, wo er an der Gründung des ersten Außenpostens der NSDAP in Südamerika mitwirkte; Anfang 1930 schufen er und Hans Nieland die Auslandsabteilung der Reichsleitung der NSDAP, aus der später die Auslandsorganisation (AO) hervorging. Von Hamburg zog er nach Danzig, wo er die lokale SA leitete und NS-Funktionär wurde. ${ }^{2}$ Seine Treue zu den Strasser-Brüdern und ihrem "linken" Nationalsozialismus verursachte im September 1930 seinen Ausschluß aus der Partei. Danach kehrte er nach Südamerika zurück.

1932 gründete Fricke lokale Ableger der "Schwarzen Front" in Paraguay, Brasilien und Argentinien. 1933 zog er nach Santiago um, wo ihn die chilenische Polizei auf Beschwerde des Ministers Kaufmann in Buenos Aires für kurze Zeit festhielt. Viele unzufriedene Auslandsdeutsche gingen nach dem Juni 1934 von der NSDAP zur "Schwarzen Front" über; kleine Zellen bildeten sich in ganz Südamerika. Fricke hielt sich sodann in Paraguay auf: Er und seine Frau reisten auf dem Pferderücken zwischen verstreuten deutschen Siedlungen umher und machten Propaganda. Von seiner Estancia in der deutschen Kolonie in Encarnación aus leitete er alle Operationen der "Schwarzen Front" auf dem Kontinent. Gegen Ende 1935 verlegte er erneut seinen Wohnsitz, diesmal nach

${ }^{1}$ Eine Ausnahme bildete hierbei die Freundschaft zwischen dem Berliner Ernst Damerau, einem Sozialdemokraten, und dem amerikanischen Botschafter Norman Armour. Diese Beziehung war in der Öffentlichkeit kaum bekannt - wohl in beider Interesse.

${ }^{2}$ Das Andere Deutschland (DAD) 47 (Febr. 1942): 1-3. 
Buenos Aires, dem größten Zentrum radikaler Ex-Nazis in Südamerika. Dort sollte er bleiben, bis er im Januar 1943 aufgrund von möglicherweise gefälschten Belastungsmaterialien wegen Drogenhandels eingesperrt wurde. ${ }^{3}$

In Buenos Aires beschmutzte sich Fricke in dem trüben Wasser der Exilpolitik. Sein Anspruch, die Überreste des sozialdemokratischen "Reichsbanners" übernommen zu haben, fand keine Bestätigung. Jedoch brachte er - nach seiner Ausdrucksweise - "Massenversammlungen" zustande, obwohl die Gestapo, die seine Aktivitäten genau überwachte, im April 1936 die Zahl seiner Anhänger in Argentinien auf höchstens 70 schätzte. ${ }^{4}$ Dafür aber schuf er eine Organisation dieses Charakters auf dem ganzen Kontinent: Im folgenden Monat ernannte er Landesleiter für Argentinien (Helmut Gastel) ${ }^{5}$, Brasilien (Herbert Guss, einen ehemals nationalsozialistischen Zahnarzt), Paraguay, Uruguay und Chile (den Arzt und ehemaligen Nazi Theo Fuchs, einen aktiven Mitarbeiter, der Anfang 1942 aus der Bewegung ausgeschlossen wurde). In Paraguay und Chile gab es vielfältige Beziehungen zwischen "Schwarzer Front" und sozialistischen Exilgruppen.

Die Propaganda der "Schwarzen Front" erregte die loyalen Hitler-Anhänger. Fricke und Dr. Schoenemann verwandelten das Deutsche Demokratische Wochenblatt, das Schoenemann in Montevideo gegründet hatte, in eine Zweiwochenschrift Die Schwarze Front (einige nach Deutschland gelangte Exemplare sind noch erhalten) und verbreiteten das Wochenblatt Die Deutsche Revolution über ganz Südamerika. ${ }^{6}$ Gegen Ende 1935 betrieb Frickes Organisation zwei Rundfunksender, einen Kurzwellensender namens "Pampero" in

\footnotetext{
${ }^{3}$ Bruno Fricke: "Rechenschaftsbericht der FDB für die Jahre 1943 bis 1945 "; Institut für Zeitgeschichte (IfZ): ED 118, Bd. 20.

${ }^{4}$ [Schwarze Front:] Orga-Rundschau, März 1936; IfZ: ED 118, Bd. 20. - Bericht Erich Heberleins vom 15.4.1936 an die Gestapo; Politisches Archiv des Auswärtigen Amts (PA AA): Inland II A/B (83-45A), Bd. 1.

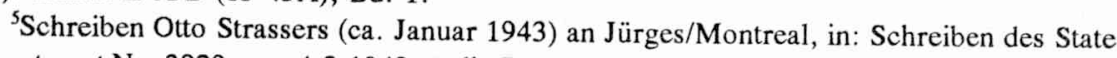
Department Nr. 3829 vom 4.2.1943 an die Botschaft in Buenos Aires; US National Archives (Suitland MD) [USNA (S)]: Record Group (RG)G 84, Buenos Aires Post Records (BAPR) 1943, Box 34, Akte 820.02. - Strasser hatte Anfang 1936 erfahren, daß die NSDAP oder die Gestapo die "Schwarze Front" unterwandert hatten.

${ }^{6} \mathrm{Nach}$ dem Scheitern der Schwarzen Front begründete Schoenemann das Blatt neu unter dem Namen Die Zeit/Montevideo. Er erpreßte von deutschen Flüchtlingen Geld mit der Drohung, daß er sie in der Zeitschrift "bloßstellen" würde, und verkaufte ihnen Bescheinigungen über ihre NS-feindliche Gesinnung. In dieser ganzen Zeit behauptete er, mit den für die "Schwarzen Listen" zuständigen Dienststellen der Alliierten in Verbindung zu stehen und preßte auf diese Weise Geld aus deutschen Firmen heraus. Vgl. Das Andere Deutschland (November 1941, 44). - Schreiben der US-Botschaft Montevideo Nr. 76 vom 20.8.1941 an das State Department, sowie Nr. 1770 vom 31.10.1942; USNA: RG 59, 862.20210/561 bzw. 862.20210/2016.5. - Fernschreiben Schoenemanns vom 24.3.1943 an Otto Strasser über Geheimdienstoffiziere in San Juan/Puerto Rico R-219-43; USNA: RG 38, C-10J-23106B. Weitere biographische Daten zu Schoenemann s. von zur Mühlen 1985, 147-149.
} 
Richtung Deutschland und einen Langwellensender namens "Lasso" mit Sendungen für die Deutschen in Südamerika. Das Reichsluftfahrtministerium teilte dem Propagandaministerium mit, daß Frickes dreimal wöchentlich ausgestrahlte Sendungen zu schwach und amateurhaft seien, um in Deutschland abgehört zu werden, jedoch wurde ihm auf Köhns Empfehlung 1936 die deutsche Staatsbürgerschaft entzogen. ${ }^{7} \mathrm{Zu}$ jener Zeit wurden - zumindest nach eigener Aussage - von Gestapo-Agenten mindestens zwei Attentatsversuche gegen ihn unternommen; außerdem wurde er erneut verhaftet, diesmal von den Argentiniern. ${ }^{8}$

Die größte Last bedeutete für Fricke Heinrich Jürges. Dieser hatte eine reiche kriminelle Vergangenheit hinter sich, trat 1930 der NSDAP bei und wurde 1933 ausgeschlossen. Er wanderte nach Chile aus und leitete zeitweilig eine Zelle der "Schwarzen Front" in Talca. 1936 übersiedelte er etwas übereilt nach Buenos Aires, wo er sich in Frickes Organisation einschlich und dann versuchte, sie zu übernehmen. Er verbreitete mit einem erfundenen Brief, daß Fricke nach wie vor mit den Nazis geheime Kontakte pflege; der Brief wurde am 26. April 1936 veröffentlicht. Zwei Tage später mußte Jürges die Nachricht widerrufen, - zum Schaden seines eigenen Ansehens als auch dessen Frickes (Volberg 1981, 121). Kurz darauf stahl Jürges die Kartei der "Schwarzen Front" mit den Unterlagen über Nazis und Ex-Nazis - sie sollte später wie ein Irrlicht durch die Geheimdienstkreise geistern - und krönte seinen Plünderungszug durch so gründliches Ausräumen der Parteikasse, daß das Blatt Die Schwarze Front sein Erscheinen einstellen mußte. Wie Das Andere Deutschland es formulierte: "Ein Führer betrog den anderen, so wie beide vorher ihre Anhänger." 9

Danach erfuhr Frickes Anhängerschaft einen weiteren Aderlaß. Fricke kündigte eine Redetournee durch Chile an, um die viel Aufhebens gemacht wurde; für die Kosten brachten loyale Anhänger der "Schwarzen Front" eine beachtliche Geldsumme auf, die ein gewisser "Franz Schubert" nach Buenos Aires brachte. Dort fand er nach längerer Suche Fricke in dem Gasthaus "Schwarzer Adler", das dieser kurz vorher gekauft hatte. Fricke nahm das Geld

\footnotetext{
${ }^{7} \mathrm{Am}$ 14.8.1936 teilte der deutsche Botschafter in Buenos Aires, v.Thermann, dem argentinischen Außenministerium (MRE) mit, daß Fricke und ein gewisser Erich Herrguth wegen "Vernachlässigung ihrer Pflichten gegenüber Volk und Reich" ihre deutsche Staatsangehörigkeit verloren hätten. Ministerio de Relaciones Exteriores/Archivo de la Canillería, Buenos Aires (im folgenden MRE/AC): División Política, Box 3544, Alemania 1936, 4/36.

${ }^{8}$ Fricke: "Rechenschaftsbericht" (Anm. 4). - Das Andere Deutschland (November 1941, 44): 7. - Schreiben der Gestapo (Müller) vom 8.2.1936 an das Reichsinnenministerium; Schreiben des Reichsluftfahrtministeriums/Forschungsamt vom 2. März 1936 an das Propagandaministerium; Schreiben des Auswärtigen Amts (Heberlein) vom 15.4.1936 an die Gestapo; PA AA: Inland II A/B (83-45A) Bd. 1.

${ }^{9}$ Das Andere Deutschland 1.8.1947 (Zitat) und 44 (November 1941). - Fricke: "Rechenschaftsbericht" (Anm. 4). - Heberlein (Anm. 9).
} 
und steckte es unbekümmert in die Restaurant-Kasse. Auf der Stelle trat der begriffsschnelle "Schubert", der früher einmal in Talca Jürges Geld geliehen hatte, aus der "Schwarzen Front" aus und lieferte seine Kenntnisse schleunigst an die deutsche Botschaft. ${ }^{10}$

In den späten 30er Jahren lag die "Schwarze Front" in Südamerika ziemlich darnieder, kam jedoch 1940 zu neuem Leben, als Fricke und Otto Strasser neue Energien beisteuerten. Im Mai 1933 war Otto Strasser zunächst nach Österreich, dann in die Tschechoslowakei geflüchtet. Obwohl er dem Schicksal seines Bruders Gregor, der im Verlauf des sog. Röhm-Putsches im Juli 1934 ermordet wurde, entging, wurde sein Leben weiterhin von Anschlägen der Gestapo bedroht, so daß er seine Flucht in Richtung Portugal fortsetzte. In der zweiten Jahreshälfte 1940 war er in britischem Gewahrsam auf Bermuda, von wo ihn die Briten in ein abgelegenes Exil nach Montreal brachten. Um Antifaschisten mit gutem Gedächtnis zu besänftigen, benannte Strasser die "Schwarze Front" in "Frei-Deutschland-Bewegung" (FDB) um. Zunächst von Bermuda, dann von Kanada aus versuchte er, die Kontakte zu seinen Anhängern in der deutschen Diaspora wiederherzustellen (von zur Mühlen 1985, 149f.). Obwohl die kanadischen Behörden ihm die Veröffentlichung von Büchern untersagten, durfte er - möglicherweise sogar dazu ermuntert - eine umfassende Korrespondenz führen, die von den Geheimdiensten Kanadas, der USA, Großbritanniens und Deutschlands aufmerksam beobachtet wurde. ${ }^{11}$ Die FDB hatte ihren größten Erfolg in Südamerika, wo Fricke ihr erster Vizepräsident für den Subkontinent wurde. Darüber hinaus hatte die FDB eine kleine Anhängerschaft in Südafrika; in den USA hingegen verfügte sie möglicherweise über keinen einzigen Anhänger. ${ }^{12}$

Mit glänzenden Aussichten wiedereröffnete Fricke die Geschäftsstelle der "Schwarzen Front" in Buenos Aires. Er vertraute die argentinischen Angelegenheiten dem Kaffeehaus-Kellner Walter Trenkelbach an und übertrug ihm die Koordination des ausgedehnten, überwiegend auf dem Papier existierenden FDB-Reiches sowie die Arbeit für die alliierten Geheimdienste. Glücklicherweise für Fricke begann in diesem Jahre der Parlamentsausschuß über antiargentinische Aktivitäten seine Arbeit. In der ersten Hälfte des Jahres 1942 informierte Strasser einen anderen Korrespondenzpartner darüber, daß Fricke den Ausschuß mit "80 Prozent" seiner Daten über heimliche deutsche Aktivitäten versorgt

10"Schubert": "Das Opfer eines großen Glaubens" (undatierter Anhang zu Thermanns Schreiben vom 21.10.1936 an das AA; PA AA: Inland I A/B (83-45A) Bd. 1. - Laut Thermann war der Name "Schubert" ein Pseudonym.

${ }^{11}$ Die Alliierten fingen seine Post in Kanada, Bermuda, Panama, Puerto Rico und an anderen Stellen ab, die Deutschen über die Post in Brasilien und Paraguay.

${ }^{12}$ Bernhard Strasser, der die Priesterweihen empfangen hatte und im St. John's College in Minnesota lebte, setzte sich für seinen Bruder Otto ein und wirkte als sein Treuhänder in den Vereinigten Staaten. Über Süd- und Mittelamerika vgl. von zur Mühlen 1985, 150-153. 
habe und 34 Zeugen von der "Schwarzen Front" gestellt worden seien. ${ }^{13}$ Nach Auskunft von Hugh Millard war Fricke "für eineinhalb Jahre bezahlter Agent des örtlichen britischen Geheimdienstes"; sich selbst bezeichnete er gleichermaBen als amerikanischen Agenten. ${ }^{14}$ Er erhielt zweifellos britische Unterstützung, ebenso wie Schoenemann und Fuchs. ${ }^{15}$ Die Briten beschafften ihm Arbeit in der "Acción Argentina", einer von ihnen 1940 durch einheimische Strohmänner gegründeten proalliierten Lobby. Die Nordamerikaner glaubten, daß Fricke in gleicher Weise Geld sowohl von der deutschen als auch von der britischen Botschaft empfing; jedoch vermerkten sie im Juni 1942, daß die "britischen Stellen nicht mehr bereit seien, Strasser weiter zu unterstützen". ${ }^{16}$

Millard schrieb im Mai 1943, daß drei Jahre früher "ein Botschaftsangehöriger" sich geweigert habe, durch einen deutsch-jüdischen Mittelsmann von Fricke eine Kartei mit 10.000 Namen zu kaufen, und leugnete, daß Fricke jemals für die Nordamerikaner gearbeitet habe. Es scheint aber, daß der "Rechtsattaché" (FBI) der Botschaft tatsächlich derartige Kontakte mit Fricke unterhalten und von ihm Informationen erhalten hatte. Botschafter Armour betrachtete die Strasser-Anhänger als "Opportunisten" und war skeptisch hinsichtlich ihrer Intelligenz, glaubte aber nichtsdestoweniger, daß Fricke über "nützliche nationalsozialistische Kontakte" verfügte. ${ }^{17}$ Und es ist keine Frage, daß Propagandaschriften wie Kurt Singers Germany's Secret Service in SouthAmerica (New York 1942), das Gemeinschaftswerk von Otto Strasser und Douglas Fairbanks Hitler's Shadow over South America (Brooklyn o.J., ca. 1942) und sensationelle Artikel in verbreiteten Zeitschriften wie Coronet, Liberty, Current History, Collier's und American Mercury auf Informationen des "südamerikanischen Büros der Frei-Deutschland-Bewegung" beruhten. ${ }^{18}$

\footnotetext{
${ }^{13}$ Schreiben Strassers vom 22.1.1942 an Engelhardt; IfZ: ED 118, Bd. 20.

${ }^{14}$ Schreiben der US-Botschaft Buenos Aires vom 6.5.1943 an das State Department; USNA(S): RG 84, BAPR 1943 (Confidential), Box 34, Mappe 820.02 E-1.

${ }^{15}$ Schreiben August Siemsens vom 8.4.1942 an Hans Vogel; IfZ: ED 118, Bd. 20. - Man vermutete, daß Fuchs 800 argentinische Pesos monatlich erhielt, bis die Briten entdeckten, $\mathrm{da} \beta$ er gleichzeitig nachts einen geheimen deutschen Sender betrieb.

${ }^{16}$ Vermerk vom 21.5.1942 für das State Department; USNA: River Plate Affairs (RPA), Dept. of State (DS), 862.20210/Fricke/21. - "Political Warfare Notes" vom 17.6.1942; USNA: Reports of spec. sec., Division of Republics of America (DRA), Bd. 3, 267-75, Box 13.

${ }^{17}$ Schreiben der US-Botschaft Buenos Aires vom 6.5.1943, zitiert in Note 41; "Toop Memo" vom 6.2.1942; USNA: American Republics Affairs (ARA) gen. memos, Box 6. "Not too much reliance should be placed upon information from the Strasserites", Schreiben Chapins vom 12.9.1942 an Bonsal; USNA: Toop/Chapin spec. memos, DRA gen. memos, Box 66. - Schreiben Armours vom 2.1.1942 Nr. 3780 an das State Department; USNA: RG $59,862.20235 / 715$.

${ }^{18}$ FDB: "Rundschreiben", Mai 1942; IfZ: ED 118, Bd. 20. - Singers Verwicklung wird beschrieben im Baldwin-Memorandum vom 5.2.1942; USNA: RG 59, ARA gen. memos, Box 6. - Das Vorwort des Strasser-Fairbanks-Buches gibt als Verfasser die FDB an.
} 
Strasser kämpfte von seinem Exil im großen weißen Norden aus für die Schaffung einer Bewegung ähnlich dem "Freien Frankreich", die sowohl für nicht-marxistische Deutsche als auch für die Alliierten annehmbar gewesen wäre; am Ende aber befriedigte er keine Seite. Sein Antikommunismus, meinte er, beruhe auf humanitären, nicht ökonomischen Grundsätzen; er suchte "menschliche Würde und wirtschaftliche Sicherheit", die nach seiner Überzeugung weder vom Kapitalismus noch vom Kommunismus angeboten wurden. Als eine der wenigen praktischen Maßnahmen schlug er vor, daß alle Reichsdeutschen unter alliierter Jurisdiktion ihre politische Loyalität erklären sollten. Erklärte Nazis sollten interniert werden; den Nazi-Gegnern sollte dafür ein von den Alliierten und ihm unterzeichneter $\mathrm{Pa}$ ausgehändigt werden. Nazi-Gegner sollten überdies $10 \%$ ihrer letzten Einkommenssteuer als "Kriegsanleihe" an ihr jeweiliges Aufenthaltsland zahlen. ${ }^{19}$ Ungeachtet der Verschwommenheit des FDB-Programms, nicht zu vergessen den Mangel an Aufrichtigkeit in der jüdischen Frage, erreichte Strasser doch ein gewisses $\mathrm{Maß}$ an Zusammenarbeit mit den Alliierten und einige Anhängerschaft in Südamerika. ${ }^{20}$ Das Jahr 1942 stellte in dieser Hinsicht einen Höhepunkt dar.

Amerikanische Beamte hegten keinerlei Illusionen hinsichtlich Strasser und seiner Frei-Deutschland-Bewegung. Am 18. Mai 1942 leitete William Donovan vom Geheimdienst OSS (Office for Strategic Studies) einen aus Kanada abgefangenen Brief mit der Bemerkung an Roosevelt weiter: "Ich habe die Nachricht, daß Strassers wichtigster Helfershelfer Fricke ganz offen bekennt, daß die 'Frei-Deutschland-Bewegung' ein zweckdienliches Etikett für Nationalsozialismus ist, [...] es sind Nazis ohne Hitler." ${ }^{21}$ Die Nordamerikaner waren daher unempfänglich, als Fricke gegen Ende 1942 auf die Billigung einer "Deutschen Legion" aus hitlerfeindlichen Freiwilligen (nach dem Vorbild einer vergleichbaren südafrikanischen Einheit) drängte und die amerikanischen Beamten in Buenos Aires damit behelligte, er wolle eine Genehmigung zu einer KanadaReise erhalten, um diese Fragen persönlich mit Strasser zu diskutieren.

${ }^{19}$ Otto Strasser: "Memo über die Klassifizierung der Deutschen im Ausland" vom 15.3.1942; IfZ: ED 118, Bd. 20. - Schreiben Strassers vom 5.7.1942 an Hans Pfeifer/San Salvador (den FDB-Landesführer für Mittelamerika); US-Botschaft Panama Nr. 2544 vom 9.7.1942, USNA: RG 59, 862.20210/1639.

${ }^{20}$ Im Januar 1942 sandte Fuchs einen "Rundbrief" an die FDB-Mitglieder, in dem er Strasser wegen dessen Behauptung, sich nicht bei den Alliierten anzubiedern, kritisierte. Dafür wurde Fuchs aus der Bewegung ausgeschlossen. Er und Schoenemann waren lautstarke Antisemiten. - Fricke: "Rechenschaftsbericht"; IfZ: ED 118 Bd. 20.

${ }^{21}$ Donovan Reports, folder 11; Franklin D. Roosevelt Presidential Library, Hyde Park/NY (FDRL): Presidential Secretary File 166. - Siehe auch Toop an English vom 9. März 1942, USNA: RG 59, 862-20210/Fricke/13, einschließlich des von einem enttäuschten FDB-Mitglied übermittelten Fricke-Zitats: "Ich würde das nicht in der Akte lassen, wenn ich Du wäre. Vernichte es besser, aus verschiedenen Gründen!" 
In der Tat, die Nordamerikaner hatten von Fricke die Nase voll und gingen nicht allzu feinfühlig vor, um ihn loszuwerden. Clifton English berichtete nach Washington, daß Fricke "einem Mitglied der US-Botschaft den Verkauf von Drogen angeboten habe". Er wurde von der argentinischen Polizei eingesperrt, unter Protest natürlich und seine Unschuld beteuernd. ${ }^{22}$ Bedauerlicherweise (aus der Sicht der Botschaft) wurde er auf richterliche Anordnung zwei Tage später wieder freigelassen, im Februar aber erneut festgenommen. Bei dieser Gelegenheit beschlagnahmte die Polizei drei Truhen mit Materialien der "Schwarzen Front" sowie Frickes nunmehr auf 18.000 Namen angeschwollene Nazi-Kartei für Südamerika. Er wurde für sieben Monate in Gewahrsam genommen und dann von Präsident Ramírez freigelassen. Die argentinische Polizei bot ihm (nach eigenen Angaben) Geld für die Enthüllung britischer und amerikanischer Agenten in Argentinien an, aber er widerstand mannhaft dieser Versuchung. So wurde er bis August 1944 in Santa Fé interniert. Erneut in Freiheit gesetzt, wurde ihm nachdrücklich nahegelegt, in Richtung Paraguay abzureisen. Er tat dies und landete schließlich in einer Hutterer-Siedlung am oberen Paraguay, die sich "Bruderschaft religiöser Sozialisten" nannte. Dorthin zogen sich er und seine Frau eine Zeitlang zurück, um sich "zu läutern". ${ }^{23}$ Längere Zeit nach Kriegsende, im Februar 1949, begründete er mit einigen alten Kameraden, unter ihnen Trenkelbach, Fuchs und Schoenemann, die "Schwarze Front" unter dem Namen "Deutschland-Erneuerungsbewegung" wieder. Es hieß, daß Fricke sich auf seine Rückkehr nach Deutschland vorbereite. Kurze Zeit später verwirklichte er diesen Plan. ${ }^{24}$

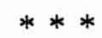

Spruille Braden war einer der ersten US-Diplomaten, die wegen der Herausforderung der amerikanischen Interessen durch die Deutschen in der westlichen Hemisphäre Alarm schlugen. Während der Friedensverhandlungen zum ChacoKrieg in Buenos Aires 1936 traf er den deutschen Flüchtling und Journalisten Tete Harens Tetens, der die Basler National-Zeitung vertrat. Tetens, von den Amerikanern "Fritz" genannt, bezeichnete sich selbst als Nachfahren eines jahrhundertealten Bauerngeschlechts aus Friesland, dem "deutschesten Teil von Deutschland". 1931, schon als Nazi-Gegner und Anti-Alldeutscher bekannt,

${ }^{22}$ Kuriermeldung von English vom 5.2.43 an Toop; USNA (S): BAPR 1943 (C), Box 34, Mappe 820.02, Argentinisches Tageblatt. Zu Fricke: JRT Memo vom 24.12.1942, USNA: RG 59, 862.20210/Fricke/47; Polutnik Memo vom 28.12.1942/48; Visa-Abteilung vom 8.1.1943; (über Puerto Rico abgefangenes) undatiertes Schreiben Frickes an Bernhard Strasser; USNA: RG 38, C-10j-23106B; Vermerk der US-Botschaft Nr. 10049 vom 6.5.1943.

${ }^{23}$ Fricke: "Rechenschaftsbericht" (Anm. 4).

${ }^{24}$ Allgemeine Kölnische Rundschau, 2.2.1949. - Nach von zur Mühlen (1985, 155) arbeitete Fricke in Deutschland für Strasser, der gleichfalls zurückgekehrt war. 
wurde er wegen einiger Artikel in Ossietzkys Weltbühne zusammen mit diesem angeklagt. Nach der nationalsozialistischen Machtergreifung wurde er im KZ Oranienburg eingesperrt, konnte aber in die Schweiz entkommen. Er schrieb dort weiterhin über die deutsche Wiederaufrüstung und Außenpolitik. Seine Artikel wurden später als Sammelband unter dem Titel Whither Hitler? veröfentlicht. Tetens Enthüllungen beeindruckten Braden so sehr, daß er dem State Department empfahl, ein "Büro für deutsche Flüchtlinge zur Berichterstattung über nationalsozialistische Literatur und Aktivitäten" einzurichten. Bradens Vorschlag wurde mit einer verhüllten Mißbilligung begrüßt, auf die solcher Übereifer gewöhnlich stößt, aber Washington erlaubte Tetens die Benutzung des diplomatischen Kurierdienstes, stellte ihm behelfsmäßige Reisedokumente aus und unterstützte möglicherweise 1937 sein Buch Christentum, Hitlerismus und Bolschewismus. ${ }^{25}$

Tetens beschaffte der amerikanischen und der britischen Botschaft in Argentinien zahlreiche Informationen - einige genaue, andere mehr phantastische, ${ }^{26}$ - und zog 1938 in die Vereinigten Staaten. Mitte 1940 - nachdem er beobachtet hatte, daß die Amerikaner auf die schlimmsten Szenarios ebenso süchtig waren wie pummelige Mädchen auf Eisportionen - diente er Staatssekretär Cordell Hull als Berater in Lateinamerika-Fragen. Er schrieb "Latin American Totalitarian Activities", eine Studie, die die Grundlage für Laurence Duggans bedeutendes Memorandum vom 10. September 1940 für Hull bildete. Nach Tetens und Duggan strebte Deutschland keine unmittelbaren territorialen Gewinne in der westlichen Hemisphäre an, vielmehr bestand die deutsche Bedrohung in 1. antiamerikanischer Propaganda, 2. wirtschaftlicher Konkurrenz, 3. Spionage, 4. nationalsozialistischen Vorbereitungen zur Unterstützung prodeutscher lokaler Regierungen oder zur Entfaltung deutscher Macht, 5. in der Ausdehnung des deutschen Flugnetzes in Südamerika, 6. in Plänen für Staatsstreiche in etlichen Ländern.

Aber der deutsche Expansionismus in Lateinamerika könne, so Tetens, durch einen deutschen Sieg in Europa beschleunigt werden. Mit den erbeuteten

\footnotetext{
${ }^{25}$ Schreiben der britischen Botschaft/Buenos Aires vom 10.2.1938 an FO; Ovey 98 vom 9.3.1938 an FO, beide: Public Record Office, London (PRO): Foreign Office (FO) $371 / 21650$.

${ }^{26} \mathrm{Er}$ beschrieb korrekt die Namen und Funktionen der deutschen Botschaftsangehörigen, des "Hafendienstes", welcher deutsche Dissidenten bei ihrer Rückkehr nach Deutschland der Gestapo übermittelte, sowie die pronazistische Gesinnung des argentinischen Militärattachés in Berlin, Oberst J.C. Sanguinetti. Er übertrieb Dietrich Niebuhrs Erfolg bei der Errichtung von Depots für deutsche Kaperaktionen zur See und in der Luft vor der argentinischen Küste - diese "La Plata-Etappe" scheint nur auf dem Papier existiert zu haben - und er wurde mißverstanden hinsichtlich der geheimen Waffenlager der HJ und ihrer Pläne, deutsche zivile Flugzeuge für militärische Zwecke zu nutzen. Ovey 89 vom 3.3.1938, PRO: FO371/21650; Schreiben Weddells Nr. 1965 vom 18.3.1938 an das State Department; USNA: RG 59, $800.20210 / 54$. FO.
} 
oder neutralisierten Flotten der Briten und Franzosen könne Deutschland in die Position gelangen, die Handelsschiffe der unterworfenen europäischen Staaten zu nutzen, um den lateinamerikanischen Seehandel zu beherrschen. Deutschland würde auf diese Weise Lateinamerikas Rohstoffindustrien an die unter deutscher Vorherrschaft reorganisierte europäische Industrie binden. Die Stellung der amerikanischen Wirtschaft würde durch deutsche Schleuderpreise für europäische Produkte auf dem lateinamerikanischen Markt schweren Schaden erleiden. Die von den Deutschen kontrollierten Luftfahrtgesellschaften würden die amerikanischen Gesellschaften verdrängen. Die Deutschen würden lateinamerikanische Partnerregime begünstigen und zum Sturz nazifeindlicher Regierungen beitragen, letztlich um prodeutsche Regime einzusetzen. Schließlich würde der amerikanische Außenhandel stranguliert werden, und ein geschwächtes Amerika stünde einem massiven Block deutscher Satelliten im Süden gegenüber. ${ }^{27}$

In seinem folgenden Memorandum "Dakar: nationalsozialistisches Sprungbrett nach Lateinamerika" schmückte Tetens dieses Szenario noch weiter aus. Darin sagte er voraus, daß "die Schlacht um die Kontinente sich nahe". Die Deutschen, schrieb er, seien dabei, Dakar zum "Gibraltar des Atlantiks" auszubauen. Hierfür bereiteten sie eine atlantische Armada von 8.000 viermotorigen Flugzeugen vor, die bis 1942 fertiggestellt sein sollten. Dies würden die Streitkräfte für eine deutsche Invasion sein, die sich der bereits eingerichteten Brükkenköpfe in Brasilien, Mexiko und Argentinien bedienen würde. Die amerikanische Verteidigung der westlichen Hemisphäre würde umgangen werden wie im Mai 1940 die Maginot-Linie. Die Vereinigten Staaten könnten dieser tödlichen Bedrohung nur durch den Ausbau ihrer eigenen Armada bis 1942 begegnen (ebda.). In seiner dringlichen Ansprache an die Vertreter des lateinamerikanischen diplomatischen Korps am 27. Mai 1941 präsentierte Roosevelt eine Version von Tetens' Szenario. ${ }^{28}$ Die meisten Hinweise des State Departments auf Tetens fehlen im National Archive, und eine genaue Bestimmung seines Einflusses auf Hull ist unmöglich. Aber nach den knappen Auskünften älterer Lateinamerika-Berater muß er beträchtlich gewesen sein. ${ }^{29}$

${ }^{27}$ Hull Papers 79/45/331, Manuskript Room; Library of Congress (LC). - Von diesen Papieren ist nur das "Dakar Memo" unterzeichnet. Der Stil ist jedoch derjenige von Tetens, und Teil 4 ("Argentina: Current and Prospective Situation") wiederholt genau die Argumente, die er schon 1938 den Briten und Nordamerikanern gegenüber vorgetragen hatte.

${ }^{28}$ Berichtet vom argentinischen Botschafter Felipe Espil: MRE/AC, DP. Guerra Europea 18-23, nicht numerierte Box, Mappe 23, Anh. 1.

${ }^{29} \mathrm{Im}$ Jahre 1943 begegnete Tetens Friedrich Wilhelm Foerster, Rex Stout, Emil Ludwig und anderen in der "Society for the Prevention of World War Three". Tetens blieb Verfechter eines harten Friedens und einer Dezentralisation in Nachkriegsdeutschland.; Memorandum vom 10.6.1944; USNA: RG 59, ARA, Div. Analysis/Liaison, Box 14. 1961 wurde in New York sein Buch The New Germany and the Old Nazis über Nazis in Argentinien veröffentlicht. 
Im Oktober 1940, kurz nach dem Duggan-Memorandum, erhielt Foggy Bottom den Besuch des "unabhängigen" österreichischen Wissenschaftlers Iso Brante Schweide. Anders als der nüchterne und pflichtbewußte Tetens war Schweide ein Flaneur, ein später Münchhausen. Er gab bescheiden zu, daß er 1917 an der Zimmerwalder Konferenz in der Schweiz teilgenommen hatte und später Lenin und den Bolschewiki nach Rußland gefolgt war. Er räumte ein, "fortschrittliche Ideen" zu vertreten, bestritt aber, Kommunist zu sein. Zwanzig Jahre lang lebte er in einem großartigen Haus in Berlin, aber 1931 riß seine Glückssträhne. In den frühen 30er Jahren lebte er in Granada, wo man ihn als "Spion" verdächtigte; er hatte via Schweden Waffen für die Spanische Republik besorgt (Fernando de los Ríos, der letzte republikanische Botschafter in den Vereinigten Staaten, verbürgte sich für ihn). Zur gleichen Zeit unterhielt er in irgendeiner Form Beziehungen zu Wilhelm und Edith Faupel im IberoAmerikanischen Institut in Berlin; Wilhelm Faupel, dem er sein Manuskript über die Absichten der Nazis in Lateinamerika gezeigt hatte, riet ihm ab, es in Deutschland zu veröffentlichen. ${ }^{30}$

In Washington enttarnte Schweide die deutschen Konsuln Weidemann in San Francisco und Scholz in Boston als aktive Meisterspione und übergab den Behörden eine - in offiziellem Sprachgebrauch - "vielsagende", 25 Punkte umfassende Anklageschrift über deutsche Aktivitäten in Argentinien. Das State Department war hoch erfreut darüber: James King beschrieb ihn als "wahre Quelle wertvoller Informationen über Deutschlands Absichten in Lateinameri$\mathrm{ka} " .{ }^{31}$ Schweide wurde für besonders glaubwürdig gehalten, weil er - obwohl früher ein scharfer Kritiker des Imperialismus - jetzt ein überzeugter Anhänger von Roosevelts "Politik der guten Nachbarschaft" war. ${ }^{32}$ Die Begeisterung des Diplomaten wurde etwas gedämpft, als die Macmillen Company mit der Begründung, Schweides Manuskript sei zu lang hinsichtlich seiner Verallgemeinerungen und zu kurz hinsichtlich der enthaltenen Fakten, es für den Druck ablehnte. Nichtsdestoweniger unterstützte das State Department Schweides Plan, nach Argentinien zurückzukehren, um seinen "Lehrauftrag an der Nationaluniversität von Buenos Aires" anzutreten und sich in den antinazistischen Kreuzzug zu stürzen.

Schweide reiste zwar in Richtung Argentinien ab, gelangte aber nicht weiter als bis Mexiko City, zumal sein Lehrauftrag in Buenos Aires gar nicht existierte. In Mexiko brütete er zusammen mit dem Philosophen José Vasconcelos, den

${ }^{30}$ Schreiben Reeds Nr. 7977 vom 31.12.1942 an das State Department; USNA: RG 59, $800.20210 /$ Schweide.

${ }^{31}$ Vermerk über das Gespräch Schweide/King vom 9.10.1940; USNA: RG 59, $862.20210 / 361$.

${ }^{32}$ Nicht unterzeichneter Vermerk vom 17.10.1940; USNA: RG 59, ARA memos, Box 17. 
er 1926 in Wien getroffen hatte, das Projekt einer noch zu gründenden proalliierten Zeitschrift aus. Um die Zeitschrift vom Stapel laufen zu lassen, brauchte er natürlich Geld. Ob Washington Schweide zu diesem Zweck finanziell unterstützte, ist nicht bekannt. Edward Trueblood von der US-Botschaft jedoch benutzte ihn für Propagandazwecke. Er gab die Nazi-Organisationen in Mexiko in der mexikanischen Presse bekannt. Als "Wissenschaftler der Nationaluniversität von Buenos Aires" hielt er mehrere nazifeindliche Vorlesungen. Schließlich gab er jedoch $\mathrm{zu}$, daß seine argentinische Professur ein reines Phantasieprodukt war und verschwand von der Bildfläche. 1949 tauchte er wieder auf - noch (oder wieder) in Mexiko, als argentinischer Staatsbürger und eifriger Propagandist für den Peronismus. Sein Anti-Imperialismus kam auch jetzt wieder zum Vorschein, und seine Attacken auf die Vereinigten Staaten waren bekannt für ihre Schärfe. ${ }^{33}$

Eine der merkwürdigsten Gestalten im Umkreis der Alliierten war der Erzpriester Aleksey Polypenko, der in seiner buntscheckigen Laufbahn Informationen an die Amerikaner, Briten, Deutschen, Italiener, Polen und Sowjets verkaufte. ${ }^{34}$ Polypenko war ein untersetzter dunkler Mann, der in geistlicher Robe umhergeisterte. Er sprach Russisch, Polnisch, Ukrainisch, Deutsch, Spanisch und Englisch ("auf eine schon recht gute Art") und wurde als "gerissener, intelligenter und energischer Redner" beschrieben. 1893 in einem Winkel der Ukraine geboren, der später an Polen fiel, und Inhaber eines polnischen Passes, war er ordinierter Priester der Griechisch-Orthodoxen Kirche. ${ }^{35} \mathrm{Er}$ heiratete und zeugte zwei Kinder; 1925 konvertierte er jedoch zum Katholizismus und beschrieb sich seitdem als "Witwer". Von 1933 bis 1937 lehrte er am St. Andreas-Gymnasium in München und ließ genießerisch durchblicken, daß er eng mit den Nazis zusammengearbeitet habe, um ihre Unterstützung für die ukrainische Unabhängigkeit zu erlangen. Er behauptete, daß er Deutschland binnen drei Tagen habe verlassen müssen ${ }^{36}$, worauf ihn die Römische Kurie nach Prag, darauf nach Argentinien versetzt habe, wo er ukrainische Emigranten betreute. In Buenos Aires wurde er nach September 1939 zeitweilig von

${ }^{33}$ Schreiben Truebloods vom 15.10.1941, vom 26.10.1942 und Schreiben Raines vom 11.2.1949 an das State Department; USNA: RG 59, 800.20210/Schweide/15. Über seinen Aufenthalt in Argentinien und seine argentinische Staatsbürgerschaft ist nichts bekannt.

${ }^{34}$ Vermerk vom 4.6.1941, BSC; USNA: RG 59, 862.20211/Polypenko/10.

${ }^{35}$ Vermerk des Sonderagenten Pasqua vom 31.8.1943 für das State Department; ebd., 30. Die Beschreibung seines Hintergrundes im Jahre 1943 bezieht sich vielleicht auf russischund ukrainisch-orthodoxe Denunziationen gegen ihn.

${ }^{36}$ Der britische Geheimdienst berichtete, daß Polypenko 1938 eine "Spionage-Schule" in München besucht habe; BSC-Vermerk: USNA: RG 59, 862.20211/Polypenko . 
seinem Sohn Igor begleitet, dem die Deutschen ein besonderes Ausreisevisum von Polen nach Kanada oder in die Vereinigten Staaten gegeben hatten.

In Buenos Aires verkehrte Polypenko mit dem dortigen Gestapo-Vertreter Christian Zinsser sowie Botschaftsrat Stephan Prinz zu Schaumburg-Lippe, einem überzeugten Nazi. Er erschlich sich zugleich das Vertrauen des amerikanischen Vizekonsuls William F. Busser, den er davon überzeugte, daß er obwohl die Deutschen ihn für seine ukrainischen Kontakte bezahlten - nicht mehr an die Aufrichtigkeit der deutschen Absichten hinsichtlich der Ukraine glaube. Sein Volk würde seiner Ansicht nach von den Polen oder den Sowjets besser behandelt. Die Vereinigten Staaten, sagte er zu Busser, seien das einzige Hindernis gegen Deutschlands "grenzenlose" Pläne in den Ländern Amerikas. Er versorgte die Nordamerikaner mit einem Kartenausschnitt mit eingezeichneten geheimen deutschen Aktivitäten am La Plata und einem genauen Bericht über seine Feinde in der von den Deutschen unterstützten lokalen Zelle des Ukrainischen Nationalkomitees. Er berichtete auch über früher weißrussische Offiziere in paraguayischen Diensten, die nach seiner Aussage höchst empfänglich waren für deutsche Pläne, das Sowjetregime zu vernichten und die zaristische Aristokratie wiederherzustellen. Er berichtete gleichermaßen, daß russische, argentinische und paraguayische "Nationalisten" sich regelmäßig mit dem deutschen "Führer" für die argentinische Provinz Formosa in einem deutschen Restaurant im Städtchen Clorinda träfen. Diese eindrucksvollen Enthüllungen brachten Polypenko eine Stelle beim "Rechtsattaché" (FBI) in Buenos Aires ein. ${ }^{37}$ Ausgestattet mit britischen und amerikanischen Reisedokumenten und Visa der polnischen Exilregierung nebst einem Zusatzvermerk von S. Pickney ("Kip") Tuck von der US-Botschaft, schiffte sich der Erzpriester mit seinem Sohn Igor im März 1941 für weitere Intrigen nach New York ein.

Mit Ausnahme von Tetens war Heinrich Jürges der Emigrant, der die tiefsten Spuren im Zeitgeschehen hinterließ. Jürges war ein armseliger Spielkartenkünstler, Veruntreuer, Erpresser, Fälscher und Dieb, dessen mäßige Talente in dieser Hinsicht sich an eine manische Wut auf das Dritte Reich klammerten, das ihn hinausgeworfen hatte, einen uncharmanten Kobold, den die alliierten Missionen bei Bedarf gebrauchten und im Regen stehen ließen, wenn sie mit ihm nichts anfangen konnten. Er hatte - mit oder ohne alliierte Duldung Talent für das Zusammenbrauen von nazifeindlichen Falschmeldungen, Erpressungen und allerlei schmutzigen Tricks, sehr zur Freude nachrichtengieriger Journalisten. Einige seiner Schöpfungen wie das Patagonia-Komplott, das große

\footnotetext{
${ }^{37}$ Schreiben des US-Generalkonsulats Nr. 374 vom 11.4.1939; 800.20210/315; Vermerk Englishs Nr. 4283 vom 2.3.1942 an die US-Botschaft; USNA: RG 59, 862.20211/Polypenko/16.
} 
Ausräuchern der Nazis und die Geschichte vom Nazi-Schatz sind ins allgemeine Geschichtsbewußtsein gedrungen.

Geboren 1898 in Barmen im Wuppertal, dem Geburtsort Friedrich Engels', diente Jürges im Ersten Weltkrieg und wurde verwundet (laut v. Thermann wurde er aufgrund seiner Wunde impotent). In den 1920er Jahren wurde er mehrfach von deutschen Gerichten wegen Veruntreuung, unerlaubten Waffenbesitzes und Erpressung verurteilt. Wegen des letztgenannten Delikts mußte er vier Jahre im Gefängnis absitzen. 1930 trat er der NSDAP in Berlin bei und war zeitweise Gehilfe von Josef Goebbels. Bald wurde er jedoch von einem Parteigericht wegen (sonst nicht näher aufgeführter) "moralischer Rechtsübertretungen" verurteilt. Nach dem Prozeß wurde er 1933 aus der Partei ausgeschlossen. In Chile arbeitete er zunächst für die dortige NS-Landesgruppe als "Stabswalt". Bevor seine Vergangenheit in Deutschland ihn wieder einholte, wechselte er zur "Schwarzen Front" über und leitete eine Zelle in Talca. Wegen Unterschlagung aus der Gruppe ausgeschlossen, ging er zum Konkurrenzunternehmen "Volksfront". Er hatte lange Zeit von seiner deutschen Frau getrennt gelebt, jedoch hatten es die beiden unterlassen, eine Scheidung zu beantragen. Für Jürges bedeutete dies kein Hindernis, sich 1934 noch einmal zu verheiraten, diesmal mit einer deutsch-schweizerischen Witwe. Sie hieß Erika Rodewald und hatte zwei Kinder. Nachdem er sie 1936 um 6.000 Pesos betrogen hatte, floh er nach Argentinien, eine unglückliche Geschäftspartnerin und zahlreiche andere Gläubiger hinter sich lassend.

In Buenos Aires schrieb Jürges zwei Artikel für die "Schwarze Front", in deren einem er im Februar 1936 die Nazis der Urheberschaft des Reichstagsbrandes beschuldigte (Jürges behauptete auch, für Marinus van der Lubbe, den geistig behinderten holländischen Kommunisten, den die Nazis wegen dieses Verbrechens hinrichten ließen, gedolmetscht zu haben). Angesichts dieses Artikels empfahl Botschafter v. Thermann, Jürges die deutsche Staatsangehörigkeit abzuerkennen. Die Gestapo untersuchte Jürges' Schriften und seinen "kriminellen und antisozialen" Charakter und setzte ihn auf die sechzehnte Ausbürgerungsliste. Die Reichsregierung zog später sein geringes Vermögen in Deutschland ein, was seinen Zorn weiter anstachelte (Rout/Bratzel 1984). ${ }^{38}$

$\mathrm{Zu}$ den anderen Gaunereien, die Jürges in Buenos Aires betrieb, gehörte auch die versuchte Erpressung einer niederländischen Firma mit Hilfe gefälschter Dokumente, aus denen hervorging, daß sie Geschäfte mit der Deutschen Arbeitsfront (DAF) gemacht habe. Etwa ab März 1937 war er Informant des

\footnotetext{
${ }^{38}$ Bericht v. Thermanns vom 21.5.1937 an das AA; PA AA: Inland II A/B (83-45A), Bd. 1. - Ausbürgerungsliste 16/11, Anzeiger Nr. 267 vom 19.11.1937, ebd., Inland II A/B (83-76). - Bericht der Gestapo vom 4.4.1939 an das AA; ebd., Büro Staatssekretär: Akten betr. Patagonien. - Rout/Bratzel liefern wenig differenzierte Details zur Biographie; Jürges' getrennt lebende Frau machte hierüber einige Aussagen von Houston/Texas aus, wohin sie 1928 ausgewandert war.
} 
sozialistischen Abgeordneten Julio Noble über Nazi-Angelegenheiten. Anfang 1938 lieferte er dem Justizminister Dr. Jorge E. Coll und dem früheren sozialistischen Angeordneten Enrique Dickmann Unterlagen über die deutschsprachigen Schulen und Vereine ${ }^{39}$. Von hier aus war es nur noch ein kleiner Schritt bis zu seinem "Hauptwerk", dem Patagonia-Komplott im Jahre 1939. ${ }^{40}$

Im Januar 1940 veröffentlichte Jürges auf eigene Rechnung den Bericht über die Selbstversenkung der "Admiral Graf Spee". Darin stellte er die These auf, daß Botschafter v. Thermann, Müller und der deutsche Gesandte in Montevideo, Otto Langmann, Kapitän Langsdorff gedrängt hätten, in See zu stechen und sich in einer selbstmörderischen letzten Schlacht den überlegenen britischen Kräften zu stellen. Die Selbstversenkung, die die Nazi-Oberen überraschte, sei dann aus Langsdorffs eigener Initiative geschehen, und Langsdorffs "Abschiedsbrief" sei eine Fälschung. ${ }^{41}$ Während des Krieges stand Jürges des öfteren in Verbindung mit der britischen Botschaft, ${ }^{42}$ war an einer Fälschung beteiligt, die zu Heinrich Volbergs Sturz führte (Volberg 1981, 132), versuchte von der Familie v. Thermann $100.000 \$$ zu erpressen ${ }^{43}$ und versah den Ausschuß über antiargentinische Aktivitäten mit Informationen. ${ }^{44} 1942$ zog er nach Montevideo, wo er den ihm seelenverwandten uruguayischen Nazi-Jäger Hugo Fernández Artucio mit Material versorgte. ${ }^{45}$ Im Oktober 1942 versuchte er, der

${ }^{39}$ Volberg 1981, 121. - Cámara de Diputados 1938, 225.

${ }^{40} \mathrm{Das}$ Patagonien-Komplott beherrschte die argentinische Öffentlichkeit im April und Mai 1939. Die Behauptungen hinsichtlich deutscher Absichten, einen patagonischen Separatismus zu ermuntern und dort einen Marionettenstaat zu errichten, waren falsch, und Präsident Ortiz wußte zweifellos darum. Nichtsdestoweniger wurden der stellvertretende NSLandesleiter Alfred Müller für einige Zeit inhaftiert, deutschsprachige Einrichtungen unter immer strengere staatliche Überwachung gestellt und das deutsche Ansehen schwer beschädigt. Die komplizierte Affäre war offensichtlich vom britischen Geheimdienst unter Mitwirkung eines deutschen Fälschers (möglicherweise Jürges selbst), argentinischer Regionalpolitiker, jüdischer Antifaschisten und amerikanischer Journalisten gelenkt worden.

41 "Die Wahrheit über die Zerstörung des Panzerschiffes "Admiral Graf Spee'", Typoskript; MRE/AC: DP, Box 4254, Alemania 1939, 9/39.

${ }^{42}$ Beispielsweise im Mai 1941; nachdem er erfahren hatte, daß Rudolf Hess von Deutschland nach Schottland geflogen war, begab er sich zu Botschafter Ovey, um ihn zu einem Brief an "meinen lieben Hess" zu überreden, damit dieser alle deutschen Entwürfe für Argentinien ausplaudere: Schreiben Oveys Nr. 370 vom 20.5.1941 an das Foreign Office; FO 371/25711.

${ }^{43} \mathrm{MRE} / \mathrm{AC}$, DP, nicht numerierte Box, Alemania y otros 1941, Mappe 641. - In seinem Brief an Baronin v. Thermann behauptete Jürges, in Verbindung mit Oberst Russell von der britischen Botschaft zu stehen. Russells Plan bestand darin, Explosionen auszulösen, die dann "geheimen deutschen Waffenverstecken" zugeschrieben würden. Er nahm auch einen Kredit auf für Volbergs Streit und um einen Skandal zu provozieren, durch den der deutsche Gesandte in Bolivien, Wendler, in Ungnade fiel.

${ }^{44}$ Ebda. - El Pampero vom 13.10.1941, in: Deutsches Auslandsinstitut 874, USNA: RG 242, T81/534/5302618.

${ }^{45}$ Fernández Artucio 1942; de Jong 1959, 113, 210-213. 
uruguayischen "Comisión Investigadora de Actividades Antinacionales" eine Fälschung über einen gewissen Alfred von Metzen und seine PropagandaAktivitäten zu verkaufen. ${ }^{46}$ Etwa zur gleichen Zeit schloß ihn Otto Strasser persönlich wegen "Fälschung von Dokumenten" aus der "Schwarzen Front" aus. ${ }^{47}$ Anfang 1944 brachte Jürges die Hellmuth-Affäre mit einer anderen Fälschung durcheinander, diesmal mit einem auf deutscher Stenographie beruhenden "Code" und einem Komplott, das auf dem damals in Montevideo gezeigten Hollywood-Film "Hangmen Also Die" beruhte. ${ }^{48}$ Seitdem genoß er keinerlei Glaubwürdigkeit mehr, weder bei britischen noch bei amerikanischen Diplomaten. Aber noch hatte er einige wenige Eisen im Feuer.

Im Juni 1940 informierte er die Briten in Buenos Aires darüber, daß ein gewisser Ernst Hoppe, ein naturalisierter Argentinier, Abwehr-Agent sei. Hoppe lebte damals in Deutschland und kehrte nicht eher als drei Jahre später zurück. Als er es im Oktober 1943 versuchte, verhafteten ihn die Briten auf einem Schiff bei Gibraltar und sperrten ihn im Lager 020 ein, einer Einrichtung für politisch prekäre Gefangene. ${ }^{49}$ Hoppe erzählte angeblich seinen Vernehmern, daß er die Anlieferung von drei Kisten aus einem U-Boot, das dort im Februar 1944 landen würde, überwachen sollte. Der Inhalt bestand aus vertraulichen Dokumenten für die deutsche Botschaft, einem Kurzwellensender sowie 2,5 Mill. \$ in Gold und Juwelen aus dem Eigentum von Nazi-Funktionären. ${ }^{50}$ Ein britischer Agent in Uruguay berichtete die Geschichte Jürges, der sie mit Ausschmückungen an Fregattenkapitän Albert Benjamin, den US-Marineattaché in Montevideo, weiterleitete. Benjamin nahm sie ebenso begierig auf wie Unterstaatssekretär William Dawson in Washington. In Jürges' mit Details angereicherter Version, die angeblich von einem nicht genannten (und wohl nicht fingierten) Augenzeugen bestätigt werden könnte, war die Landung bereits im August 1943 erfolgt (Rout/Bratzel 1984, 618f.).

Offensichtlich mit der Absicht, auf deutscher Seite eine Reaktion auf die Nachricht auszulösen, daß ihre Führer sich in Sicherheit brachten, wurde dieses Lügenmärchen erstmals im Londoner Evening Standard vom 24. April 1943 in Umlauf gebracht. Jürges' Enthüllungen von Anfang 1944 banden den Nord-

\footnotetext{
${ }^{46}$ Berichte der US-Botschaft Montevideo Nrn. 1700 und 1742 vom 14. und 24.10.1942 an das State Department; USNA: RG 59, 862.20210/Jürges/1 und 2.

${ }^{47}$ Undatiertes Schreiben Strassers an Jürges, abgefangen über die US-Botschaft in Buenos Aires, vom 4.2.1943; USNA (S): RG 84, BAPR 1943, Box 34, Mappe 820.02.

${ }^{48}$ Rout/Bratzel 1984, 618. - Deutsche La Plata Zeitung, 23. und 26.1.1944.

${ }^{49}$ Forschern wird der Zugang zu Hoppes Dossier im Public Record Office, London (PRO), das zusammen mit dem von Osmar Hellmuth aufbewahrt wird, verweigert; FO371/37694B(1944); Hellmuth wurde gleichfalls im Lager 020 eingesperrt.

${ }^{50}$ FBI: "German Espionage in Latin America" (Washington 1946), 191f; USNA: RG 319, "P" file. War Dept., Military Intelligence Division, "Axis Espionage and Propaganda in Latin America" (Washington 1946); darin wird Hoppe (62) als SD-Agent bezeichnet; USNA: RG 165, Box 982.
} 
amerikanern den Bären auf, daß dies bereits geschehen sei und sich auch wiederholen könne. Als das Informationsministerium seine Desinformationskampagne im September 1944 wieder aufnahm, wandten sie sich erneut an Jürges mit der Anregung, diese Nachrichten zu verbreiten. Dies tat er, indem er in die US-Botschaft in Montevideo hineinplatzte und Märchen über die Umstände der kürzlich erfolgten Fluchtbewegung von Nazis nach Südamerika ausposaunte. ${ }^{51}$ Obwohl die meisten amerikanischen Diplomaten von nun an Jürges gegenüber skeptisch waren ${ }^{52}$ überwältigte die Raffinesse, mit der die Briten die Geschichte vom großen Nazi-Popanz garnierten, das offizielle Washington. Die Nordamerikaner bemerkten die Wahrheit erst im April 1945. Als es soweit war, vertuschten die Alliierten mit Schamesröte diese Erkenntnis (Newton 1984, 81-103)..$^{53}$

Ein beunruhigendes Problem blieb für Jürges bestehen: Hoppe. Als er im Herbst 1945 von der Freilassung Hoppes durch die Briten und seiner bevorstehenden Rückkehr nach Argentinien - sicher mit höchst unfreundlichen Gedanken - erfuhr, wuchs seine Vorstellung zum Gefühl der Bedrohung. Als er zur selben Zeit erfuhr, daß der frühere Botschafter v. Thermann möglicherweise als Kriegsverbrecher angeklagt würde, erklärte sich Jürges freiwillig bereit, gegen ihn auszusagen, besonders hinsichtlich dessen, was er über die Ermordung eines gewissen Hans Wachsel 1939 in Buenos Aires wußte. ${ }^{54}$ Die Alliierten lehnten $a b$, so daß Thermann nicht angeklagt wurde, arrangierten aber für Jürges die Rückkehr nach Deutschland im Juli 1946 an Bord des Schiffes Marine Marlin. Das Leben im zerstörten Nachkriegsdeutschland erschien ihm aber nicht angemessen, und so wandte er sich mit einer Nachricht an die Nordamerikaner, zu deren Bestätigung er (auf amerikanische Kosten) nach Argentinien zurückkehren müsse. Er war die Quelle für die Geschichte, daß Martin Bormann sich in Rio Negro versteckt halte. ${ }^{55}$ Er entwarf auch eine detaillierte Geschichte über den nach dem Kriege nach Argentinien verschobenen Nazi-Schatz, von dem ein Teil sich in den Händen Peróns befinde. Daher beauftragte Washington seine Botschaft in Buenos Aires mit einer entsprechenden Untersuchung. Aber Botschaftsrat Sidney O'Donoghue antwortete entschlossen: "Der Charakter des Informanten und die sehr geringe Möglichkeit,

${ }^{51}$ Telegraph. Vermerk Nr. 908 der US-Botschaft Montevideo vom 26.9.1944 an das State Department; USNA: RG 59, 862.20235/date. date.

${ }^{52}$ Meldung der US-Botschaft Nr. 16737 vom 29.11.1944; USNA: RG 59, 862.20235/6

${ }^{53}$ Das Schlüsseldokument ist das Schreiben der US-Botschaft vom 20.4.1945 an das Foreign Office; PRO: FO371/46766.

${ }^{54}$ Schreiben der US-Botschaft Nr. 6744 vom 21.2.1945 an das State Department; USNA: RG 59, 862.20233/date.

${ }^{55}$ Vermerk des Rechtsattachés Oglesby vom 2.1.1947 für den Botschafter, Anl. 1 zum Bericht der Botschaft Nr. 101 vom 4.1.1947 an das State Department; USNA: RG 59, 862.20210/Jürges. 
daß die Behauptungen irgendeine reale Grundlage haben, rechtfertigen es nicht, ein solches Projekt überhaupt in Erwägung zu ziehen" - und lehnte ab. ${ }^{56}$

Mit der Verpflichtung, in Deutschland zu bleiben, zog Jürges Anfang 1950 nach Ost-Berlin um und arbeitete für die DDR-Regierung. Dieser lieferte er die Namen der meist sozialdemokratischen Delegierten auf dem großen antifaschistischen Kongreß in Montevideo 1943. ${ }^{57}$ Er benutzte auch die Bibliothek des Ibero-Amerikanischen Instituts, um weitere Fälschungen vorzubereiten. Diese bestanden aus Dokumenten, die angeblich 1946 in der Reichskanzlei gefunden worden waren (er behauptete, zwei alliierte Forscher bei ihrer Suche unterstützt zu haben), sowie aus Briefen aus dem persönlichen Dossier von Wilhelm Faupel, dem letzten Direktor des Ibero-Amerikanischen Institut im Kriege (der im Mai 1945 Selbstmord begangen hatte). Diese "Dokumente" förderten ausgedehnte Beziehungen zwischen den Nazis und Juan Perón zutage und wurden in Silvano Santanders antiperonistischer Schrift Técnica de una traición: Juan D. Perón y Eva Duarte, agentes del nazismo en la Argentina im uruguayischen Exil 1951 veröffentlicht. ${ }^{58}$ Es ist unbekannt, wie lange er in Ostdeutschland blieb. 1962 wurde er jedoch in Bonn wegen Urkundenfälschung, Bigamie und nicht näher aufgeführter Schwindeleien angeklagt. Während der Prozeß noch anhängig war, veröffentlichte er in der Kölnischen Rundschau den Vorwurf, daß Mitglieder der NSDAP-Landesgruppe Argentinien den Handelsattaché Richard Burmeister 1944 ermordet hätten und daß ein gewisser Gottfried Sandstede Nazi-Gegner gewaltsam aus Argentinien entführt habe. Aufgefordert, seine Behauptungen zu belegen, mußte er passen. Die Tatsache, daß Sandstede 1944 offensichtlich getötet worden war, rettete ihn vor einer Verleumdungsklage. Aufgrund seines schlechten Gesundheitszustandes und seines allgemeinen Verfalls wurde der Prozeß 1966 eingestellt (Volberg 1981, 121f.).

Aus dem Englischen von Patrik von zur Mühlen

${ }^{56}$ Bericht der US-Botschaft Buenos Aires Nr. 2419 vom 2.5.1947; USNA: RG 59, $862.20210 /$ Jürges.

${ }^{57}$ IML/ZPA 6506. - Ich danke Wolfgang Kießling für die Überlassung seiner Notizen.

${ }^{58}$ Später wurde der Mitarbeiterstab des Ibero-Amerikanischen Instituts über die betroffenen Argentinier und über die Echtheit der Dokumente befragt. Man bezeichnete sie als raffinierte Fälschung. Ich danke Dr. Günther Vollmer vom IAI für die Überlassung von Materialien zu dieser Affäre. 


\section{Bibliographie}

Fernández Artucio, Hugo. 1942. The Nazi Underground in South America. New York: Farrar \& Rinehart.

Jong, Louis de. 1959. Die deutsche fünfte Kolonne im Zweiten Weltkrieg. Stuttgart: Deutsche Verlagsanstalt.

Mühlen, Patrik von zur. 1985. Der "Gegen-Führer" im Exil: Die Otto-StrasserBewegung in Lateinamerika. In: Exilforschung. Ein internationales Jahrbuch. III: Gedanken an Deutschland im Exil und andere Themen. München: Ed. Text und Kritik.

Newton, Ronald C. 1984. The United States, the German-Argentines, and the Myth of the Fourth Reich, 1943-1947. In: HAHR 64/1: 81-103.

- . 1992. The 'Nazi Menace' in Argentina, 1931-1947. Stanford/CA: Stanford University Press.

Rout, Leslie B., Jr. \& John F. Bratzel. 1984. Heinrich Jürges and the Cult of Disinformation. In: International History Review 6: 611-623.

Volberg, Heinrich. 1981. Auslandsdeutschtum und Drittes Reich: der Fall Argentinien. Köln/Wien: Böhlau. 